\title{
Clinicopathologic implications of tissue inhibitor of metalloproteinase-1-positive diffuse large B-cell lymphoma
}

\author{
Jung-Woo Choi ${ }^{1}$, Jung-Suk An ${ }^{1}$, Ju-Han Lee ${ }^{1}$, Eung-Seok Lee ${ }^{1}$, Kwang Hee Kim² and \\ Young-Sik Kim ${ }^{1}$
}

${ }^{1}$ Department of Pathology and Biostatistics Interest Group, Korea University Ansan Hospital, Ansan, Republic of Korea and ${ }^{2}$ Department of Medicine, Mount Sinai School of Medicine, New York, NY, USA

\begin{abstract}
The tissue inhibitor of metalloproteinase-1 (TIMP-1) is a stromal factor that promotes plasmablastic differentiation, and the survival of germinal center B-cells. The expression of TIMP-1 is known to be correlated with a subset of non-Hodgkin lymphoma at the mRNA level, and Epstein-Barr virus infection in vitro. To characterize TIMP-1(+) diffuse large B-cell lymphoma, TIMP-1 expression was investigated in tissue microarrays from 182 cases of de novo diffuse large B-cell lymphoma and compared with prognostic factors, immunophenotypes, and Epstein-Barr virus infection status. TIMP-1 was expressed not only in tumor cells themselves, in 14 of 182 cases (8\%), designated as TIMP-1(+) diffuse large B-cell lymphoma, but also in stromal cells like fibroblasts and endothelial cells. In univariate analysis and hierarchical clustering, our findings suggest that TIMP-1 expression may represent a distinct subgroup. In multivariate analysis, TIMP-1( + ) diffuse large B-cell lymphoma $(n=14)$ was associated with unfavorable outcomes compared to TIMP-1(-) diffuse large B-cell lymphoma $(n=168)$ (odds ratio $=2.5, P=0.049)$. Together with TIMP-1 expression, age (greater than 60 years), the presence of B-symptoms, abnormal lactate dehydrogenase level, or more advanced stage (III/IV) was correlated with a poor overall survival. However, TIMP-1 expression in diffuse large B-cell lymphoma was not correlated with other prognostic factors including: clinical stage, international prognostic index score, and nongerminal center B-cell phenotype, as well as Epstein-Barr virus infection. Our results suggest that TIMP-1 expression may be an independent negative prognostic factor in patients with diffuse large B-cell lymphoma. Modern Pathology (2006) 19, 963-973. doi:10.1038/modpathol.3800615; published online 28 April 2006
\end{abstract}

Keywords: TIMP-1; non-Hodgkin lymphoma; tissue microarray

The tissue inhibitor of metalloproteinase-1 (TIMP-1) is a stromal factor with multiple functions that affect cell survival and differentiation, ${ }^{1}$ as well as matrix metalloproteinase (MMP) inhibition. ${ }^{2}$ TIMP-1 inhibits germinal center B-cell apoptosis and promotes B cell survival by upregulating BCL- $\mathrm{X}_{\mathrm{L}}$ and by activating the interleukins/signal transducer and activator of transcription 3 (STAT3) signaling pathway. A cDNA microarray analysis using TIMP-1 overexpressing Burkitt lymphoma (BL) cell lines revealed that TIMP-1 promotes post-germinal center B-cell differentiation by up-regulating MUM-1 and CD138 and down-regulating BCL6. ${ }^{3}$ TIMP-1 expression has

Correspondence: Dr Y-S Kim, MD, PhD, Department of Pathology, Korea University Ansan Hospital, 516, Gojan-1 Dong, Danwon$\mathrm{Gu}$, Ansan-Si, Gyeonggi-Do 425-707, Republic of Korea.

E-mail: apysk@korea.ac.kr

Received 22 February 2006; revised 27 March 2006; accepted 1 April 2006; published online 28 April 2006 been reported in a subset of B-cell lymphoma at the mRNA level ${ }^{4,5}$ and has been shown to be associated with a poor clinical outcome in a variety of carcinomas. $^{6,7}$ Moreover, TIMP-1 overexpression has been observed in Epstein-Barr virus (EBV) positive lymphoma cell lines. ${ }^{8}$

Diffuse large B-cell lymphoma accounts for $30-40 \%$ of all new diagnoses of non-Hodgkin lymphoma. ${ }^{9,10}$ However, diffuse large B-cell lymphoma is heterogeneous with regard to natural history, clinicopathologic presentation, response to therapy, and host immune function. ${ }^{11}$ Recent cDNA microarray studies have shown that diffuse large B-cell lymphoma can be divided into four distinct subgroups including: germinal center B-cell-like, activated B-cell-like, primary mediastinal, and unclassified gene expression profiles. ${ }^{12-15}$ The activated B-cell and unclassified groups have a significantly inferior clinical outcome compared to the germinal center B-cell and primary mediastinal 
Table 1 Antibodies used for immunohistochemistry

\begin{tabular}{|c|c|c|c|c|}
\hline Antibody & Clone & Source & Antigen retrieval & Dilution \\
\hline CD10 & $56 C 6$ & Novocastra, UK & EDTA mw 10 & $1: 50$ \\
\hline BCL6 & PG-B6p & Dako, Denmark & EDTA mw 10 & $1: 10$ \\
\hline MUM1 & MUM1p & Dako, Denmark & EDTA mw 10 & $1: 25$ \\
\hline CD138 & MI15 & Dako, Denmark & EDTA pc 10 & $1: 50$ \\
\hline TIMP-1 & 102D1 & Lab vision, CA, USA & EDTA pc 2 & $1: 50$ \\
\hline
\end{tabular}

EDTA mw or pc 10 indicates pretreatment in a microwave oven or pressure cooker with EDTA (1 mM, pH 8.0) for $10 \mathrm{~min}$.

groups. The unclassified group is heterogeneous and not well defined, but has a poor outcome similar to that of the activated B-cell group. ${ }^{12-15}$ In addition, although the clinical significance of EBV infection in diffuse large B-cell lymphoma is controversial, about $10 \%$ of diffuse large B-cell lymphoma is known to be associated with EBV. ${ }^{16,17}$

However, TIMP-1 expression in diffuse large B-cell lymphoma has not been investigated using a large scale of clinical samples or at the protein level. In addition, the association of TIMP-1 expression with other clinicopathologic factors or EBV infection status has not been studied in vivo. Thus, in order to further characterize TIMP-1 $(+)$ diffuse large B-cell lymphoma, we investigated the relationship of TIMP-1 expression with other clinical prognostic and immunophenotypic markers and EBV infection using tissue microarray blocks from 182 cases of de novo diffuse large B-cell lymphoma.

\section{Materials and methods}

\section{Patients}

We retrospectively studied 182 de novo diffuse large B-cell lymphoma cases at Korea University Hospitals from 1994 to 2005. Cases with a history of AIDS, receiving immunosuppressive therapy, or a preceding diagnosis of low-grade lymphoma were excluded. All cases were reviewed to confirm the diagnosis according to the criteria of the World Health Organization (WHO) classification of lymphoid neoplasm. ${ }^{18}$ Each patient received an anthracycline-containing chemotherapy regimen. No patient received rituximab. The end point of clinical follow-up was either the date of the last contact or the date of death.

\section{Tissue Microarray Construction}

We reviewed all the hematoxylin and eosin-stained sections from each paraffin-embedded, formalinfixed block to identify diagnostic areas. Two to four representative $1 \mathrm{~mm}$ cores were obtained from each case and inserted in a grid pattern into a recipient paraffin block using a tissue arrayer (Beecher Instruments Inc., Sun Prairie, WI, USA).

\section{Immunohistochemistry}

Immunohistochemistry was performed using a ChemMate EnVision Kit (Dako, Carpinteria, CA, USA). Tissue sections were deparaffinized and dehydrated. Sections in $1 \mathrm{mM}$ EDTA buffer ( $\mathrm{pH}$ 8.0) were heated in a microwave or pressure cooker for 2-10 min. After incubation with $0.3 \% \quad \mathrm{H}_{2} \mathrm{O}_{2} /$ methanol for $20 \mathrm{~min}$, slides were stained with antiTIMP-1, CD10, BCL6, MUM-1, and CD138 antibodies (Table 1). After incubation with secondary antibody at room temperature for $30 \mathrm{~min}$, the sections were developed with $3,3^{\prime}$-diaminobenzidine, and counterstained with Harris hematoxylin.

\section{EBER-In Situ Hybridization}

EBER-in situ hybridization was carried out according to the manufacturer's protocol (Dako). The sections were digested with $10 \mu \mathrm{g} / \mathrm{ml}$ of proteinase $\mathrm{K}$ at $37^{\circ} \mathrm{C}$ for $30 \mathrm{~min}$, washed in distilled water, and then dehydrated in ethanol and air dried. Hybridization was carried out with a fluorescein-conjugated EBER peptide nucleic acid (PNA) probe (Dako, Carpinteria, CA, USA) in a humidified chamber at $55^{\circ} \mathrm{C}$ for $90 \mathrm{~min}$ and further incubated in a preheated stringent washing solution for $25 \mathrm{~min}$. The sections were treated with anti-FITC-alkaline phosphatase conjugate at room temperature for $30 \mathrm{~min}$ and washed with Tris-buffered solution and distilled water. The sections were then incubated with a substrate solution of nitroblue tetrazolium, 5-bromo4-chloro-3-indolylphosphate, and levamisole for 60-90 min. The slides were washed, stained with nuclear fast red, and mounted.

\section{Interpretation and Hierarchical Cluster Analysis of Tissue Microarray Data}

Each core was evaluated by visual estimation for the percentage of positively stained tumor cells. For each case, the core with the highest percentage of tumor cells stained was used for analysis. Tumor cells that showed distinct nuclear or cytoplasmic staining were considered positive. Cases were considered positive if $30 \%$ or more of the tumor cells were stained with an antibody other than antiTIMP-1 antibody. Based on previous immunohisto- 
chemical studies, ${ }^{19-22}$ we chose a uniform cutoff of $30 \%$. However, for TIMP-1, a cutoff of greater than $20 \%$ was used because its secretory properties and low level of expression within cells. ${ }^{23}$ The intensity of staining was also evaluated, but was not used to determine positivity because the variability in tissue fixation and processing appeared to affect the intensity of staining. Therefore, results of immunostaining and EBER-in situ hybridization were graded on a four-tiered scale (strong positive: if $50 \%$ or more of the tumor cells were stained; weak positive: 30-49\%; equivocal: 5-29\%; negative: less than 5\%). Immunostaining results of TIMP-1 were also graded as follows (strong positive: $30 \%$ or more; weak positive: $20-29 \%$; equivocal: $5-19 \%$; negative: less than $5 \%$ ).

Each subtype of diffuse large B-cell lymphoma was determined by immunoperoxidase results for CD10, BCL6, and MUM-1. ${ }^{24}$ Although four subtypes of diffuse large B-cell lymphoma were classified by cDNA microarray, the unclassified group was found to be heterogeneous and behaved in a manner similar to the activated B-cell group and the primary mediastinal group was found to characteristically develop from the mediastinum and was associated with a favorable prognosis. ${ }^{13,14}$ Therefore, we classified diffuse large B-cell lymphoma cases into two groups: germinal center B-cell or nongerminal center $\mathrm{B}$-cell group. The tissue microarray classification predicts survival similar to the cDNA microarray analysis. $^{24}$ Cases were assigned to the germinal center B-cell group if CD10 alone was positive or if both CD10 and BCL6 were positive. Since the immunostaining of MUM-1 had a tendency to be overstained, cases were assigned to the germinal center B-cell group if both CD10 and MUM1 were positive or if all three markers including CD10, BCL6, and MUM1 were positive. ${ }^{24}$ If both CD10 and BCL6 were negative, the case was assigned to the nongerminal center B-cell subgroup. If CD10 was negative and BCL6 was positive, the expression of MUM-1 determined the group: if MUM-1 was negative, the case was assigned to the germinal center B-cell group; if MUM-1 was positive, the case was assigned to the nongerminal center B-cell group.

For hierarchical clustering, immunostaining results were recorded in an Excel workbook and reformatted with Tissue Microarray-Deconvoluter version 1.06. ${ }^{25}$ Unsupervised hierarchical clustering analysis, with an average linkage algorithm, was performed using Cluster software, and the clustered output was viewed using TreeView, which graphically displays relatedness in both dimensions as a dendrogram. ${ }^{26}$

\section{Statistical Analysis}

Chi-square or Fisher exact tests were used to test the strength of the association between clinicopatho- logic parameters and TIMP-1 expression. Univariate and multivariate survival analyses were performed with the Kaplan-Meier method with log-rank tests and the Cox proportional hazard model, respectively. A $P$-value less than 0.05 was regarded as significant. Statistical analyses were carried out with the SPSS software package (version 10, SPSS Inc., Chicago, IL, USA).

\section{Results}

\section{Clinical Characteristics of TIMP-1(+) Diffuse Large B-Cell Lymphomas}

Comparison of clinical characteristics between TIMP-1(+) and TIMP-1(-) diffuse large B-cell lymphoma cases are shown in Table 2 . The patients in our study were 115 men and 67 women with a median age of 59 years, ranging from 14 to 84 years. TIMP-1 was expressed in lymphoma cells themselves in 14 of 182 cases (8\%). TIMP-1 expression was graded as strong positive, weak positive, equivocal, and negative, but all TIMP-1 $(+)$ cases were scored as strong positive. The patients with TIMP-1 expression were 10 men and four women with a median age of 61 years, ranging from 40 to 74 years. Eight patients were older than 60 years. None of the patients with TIMP-1(+) diffuse large B-cell lymphoma showed a significant association

Table 2 Clinical and immunophenotyic data

\begin{tabular}{|c|c|c|c|}
\hline Variables & $\begin{array}{c}\text { TIMP- } 1^{+} \\
\text {DLBCL }(\mathrm{n}=14) \\
\text { No. }(\%)\end{array}$ & $\begin{array}{c}\text { TIMP-1- } \\
\text { DLBCL }(\mathrm{n}=168) \\
\text { No. }(\%)\end{array}$ & $\mathrm{P}$ \\
\hline \multicolumn{4}{|l|}{ Age at diagnosis, years } \\
\hline Median (range) & $61(40-74)$ & $59(14-84)$ & \\
\hline Older than 60 & $8(57)$ & $82(49)$ & 0.549 \\
\hline Sex (male/female) & $10 / 4$ & $105 / 63$ & 0.506 \\
\hline Performance status $>1$ & 0 & $9(5)$ & 0.627 \\
\hline Serum LDH > normal & $3(21)$ & $48(29)$ & 0.567 \\
\hline Extranodal site & $13(93)$ & $131(78)$ & 0.188 \\
\hline \multicolumn{4}{|l|}{ IPI risk group } \\
\hline Low, $0-2$ & $11(79)$ & $131(78)$ & 0.959 \\
\hline High, 3-5 & $3(21)$ & $37(22)$ & \\
\hline B symptom present & $4(29)$ & $61(36)$ & 0.562 \\
\hline \multicolumn{4}{|l|}{ Stage } \\
\hline I-II & $11(79)$ & $118(70)$ & 0.51 \\
\hline III-IV & $3(21)$ & $50(30)$ & \\
\hline \multicolumn{4}{|l|}{ Subgroup } \\
\hline GC B cell & $6(43)$ & $49(29)$ & 0.284 \\
\hline Non-GC B cell & $8(57)$ & $119(71)$ & \\
\hline CD10 & $6(43)$ & $44(26)$ & 0.18 \\
\hline BCL6 & $7(50)$ & $101(60)$ & 0.459 \\
\hline MUM1 & $13(93)$ & $140(83)$ & 0.35 \\
\hline CD138 & $4(29)$ & $25(15)$ & 0.179 \\
\hline EBV & 0 & $19(10)$ & 0.184 \\
\hline
\end{tabular}


with aggressive clinical features or parameters (Table 2). None of the patients had a performance status higher than $1(0 \%, P=0.627)$, four showed $\mathrm{B}$ symptoms $(29 \% ; P=0.562)$, and three had a higher than normal serum lactate dehydrogenase (LDH) level $(21 \%, P=0.567)$. Three patients had high International Prognostic Index (IPI) scores $(21 \%$, $P=0.959)$. Although 13 cases (93\%) of TIMP-1 $(+)$ diffuse large B-cell lymphoma developed from extranodal sites, it was not significant $(P=0.188)$. Since Korea has a higher rate of extranodal lymphoma compared with other Asia countries, ${ }^{27}$ cases in the present study included an unusually high proportion of extranodal lymphomas (Table 2).

\section{Hierarchical Clustering Analysis of Tissue Microarray Data}

Hierarchical cluster analysis can sort antibodies according to similarities among the tumors that they stain. We used a correlation coefficient with centering; a similarity measure that is sensitive to the expression profile shape, regardless of the expres- sion levels. ${ }^{28}$ Antibodies clustered into groups that reflected the relatedness of expression of the cognate antigens. Interestingly, the greatest number of branches, of the antibody dendrogram, clearly separate anti-TIMP-1 from the other antibodies and EBER probe (BCL6, MUM-1, CD10, CD138, and EBER) (Figure 1). The immunostaining patterns observed for TIMP-1 were poorly correlated with those of any other antibodies in the panel, suggesting that the expression of TIMP-1 may be largely independent from that of other examined markers.

The diffuse large B-cell lymphoma cases were also subjected to a hierarchical cluster analysis, on the basis of similarity in their patterns of staining with this panel of antibodies (Figure 1). Most TIMP-1 $(+)$ diffuse large B-cell lymphoma clustered onto a single distinct branch, with high correlation, but because of the expression of CD138, two cases were clustered separately from this branch (Figure 1). Given the fact that the clustered TIMP-1 $(+)$ diffuse large B-cell lymphoma was separated from the major branches of the other cases, and that antiTIMP-1 was not correlated with the other anti- a

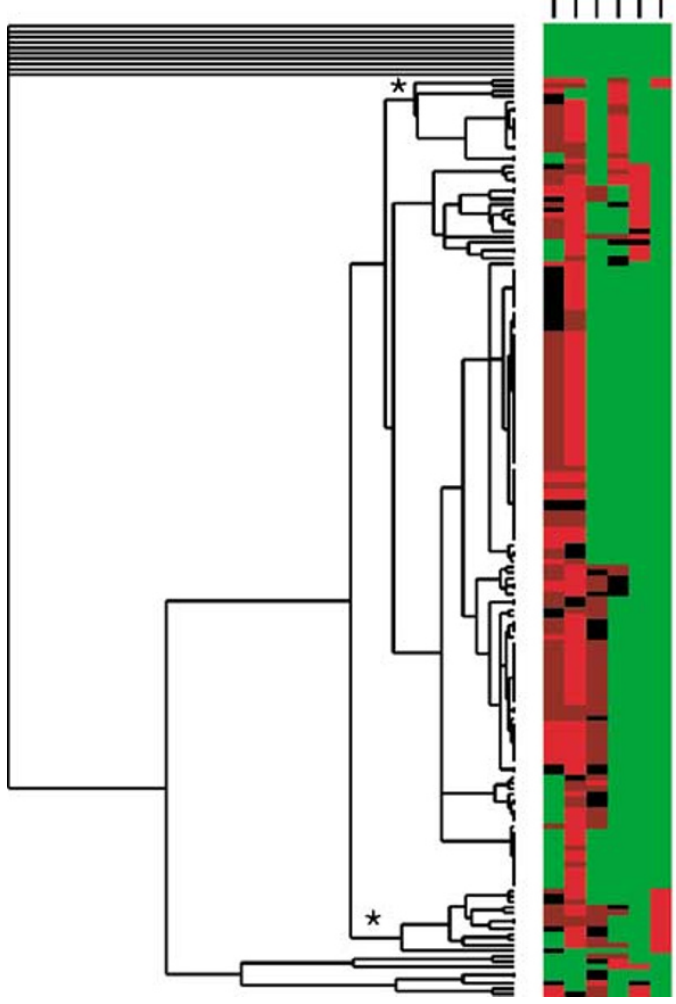

b

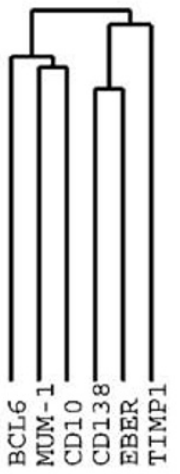

strong positive weak positive equivocal negative

Figure 1 Hierarchical cluster analysis of tissue microarray immunostaining results of diffuse large B-cell lymphoma (DLBCL). (a) Clustering result of 182 diffuse large B-cell lymphoma cases stained with five different antibodies and an EBER-in situ hybridization probe. TIMP-1 $(+)$ diffuse large B-cell lymphoma cases were clustered (asterisk). (b) For each of the antibodies, strong positive staining is indicated by a red square, weak positive as dark red, absence of staining as green and equivocal data as brown at the upper right. The dendrogram at the upper left shows the clustering of the antibodies based on the relatedness of tumors stained by each antibody. Individual case details are listed at the lower right (GCB, germinal center B-cell); the dendrogram at the lower left shows the clustering of the tumors based on the expression of TIMP-1. 
bodies, TIMP-1 $(+)$ diffuse large B-cell lymphoma may be a distinct group separate from the other cases of diffuse large B-cell lymphoma.

It was unusual that both CD10 and MUM-1 expressed in some cases, thereby leading to cluster CD10 and MUM-1 together in the dendrogram (Figure 1b). This result was probably due to overstaining of MUM-1. As mentioned earlier, the expression of CD10 determined the subgroup of diffuse large B-cell lymphoma in those cases. ${ }^{24}$

\section{Molecular Subtypes and EBV Association of TIMP-1(+) Diffuse Large B-Cell Lymphoma}

TIMP-1 was expressed in lymphoma cells themselves in 14 of 182 cases (8\%). Of the TIMP-1(+) diffuse large B-cell lymphoma, six cases were the germinal center B-cell type, while eight were the nongerminal center B-cell type. However, TIMP-1 expression was not correlated with any subtypes as defined by the tissue microarray immunohistochemistry $(P=0.284)$. Table 2 summarizes an immunophenotypic comparison of TIMP-1-positive and -negative cases. No differences in immunophenotypes were observed between the two groups. Of the 182 cases, $55(30 \%)$ were considered germinal center B-cell and $127(70 \%)$ were considered nongerminal center B-cell cases by tissue microarray analysis. Of the germinal center B-cell cases, $22 \%$ expressed CD10 alone, 7\% expressed BCL6 alone, and $71 \%$ expressed both CD10 and BCL6. Of the nongerminal center B-cell cases, 35\% expressed MUM-1 alone, 51\% expressed both MUM-1 and BCL6, and $14 \%$ were negative for all of these markers.

EBER hybridization signals were found in almost all tumor cells in 19 of 182 cases (10\%). No clinical or survival differences between EBV-positive and -negative cases were found, but $84 \%$ of EBV-positive cases were found to develop from extranodal sites such as stomach. Unexpectedly, EBV infection was not correlated with TIMP-1 expression $(P=0.184)$ (Figure 1b and Table 2).

\section{TIMP-1 Expression in Lymphoma Tumor and Stromal Cells}

TIMP-1 was also expressed in stromal cells like fibroblasts and endothelial cells lining sinusoids and capillaries in 91 of 182 diffuse large B-cell lymphoma cases (50\%) (Figure 2a). However, there were no clinical or pathologic correlates with TIMP1 expression in stromal cells. TIMP-1 was localized in the cytoplasm of the tumor cells with a Golgi staining pattern. Interestingly, the tumor cells with anaplasia or multinucleate cells frequently expressed a high level of TIMP-1 (Figure 2b). However, nuclear localization of TIMP-1 in the tumor cells was not seen.
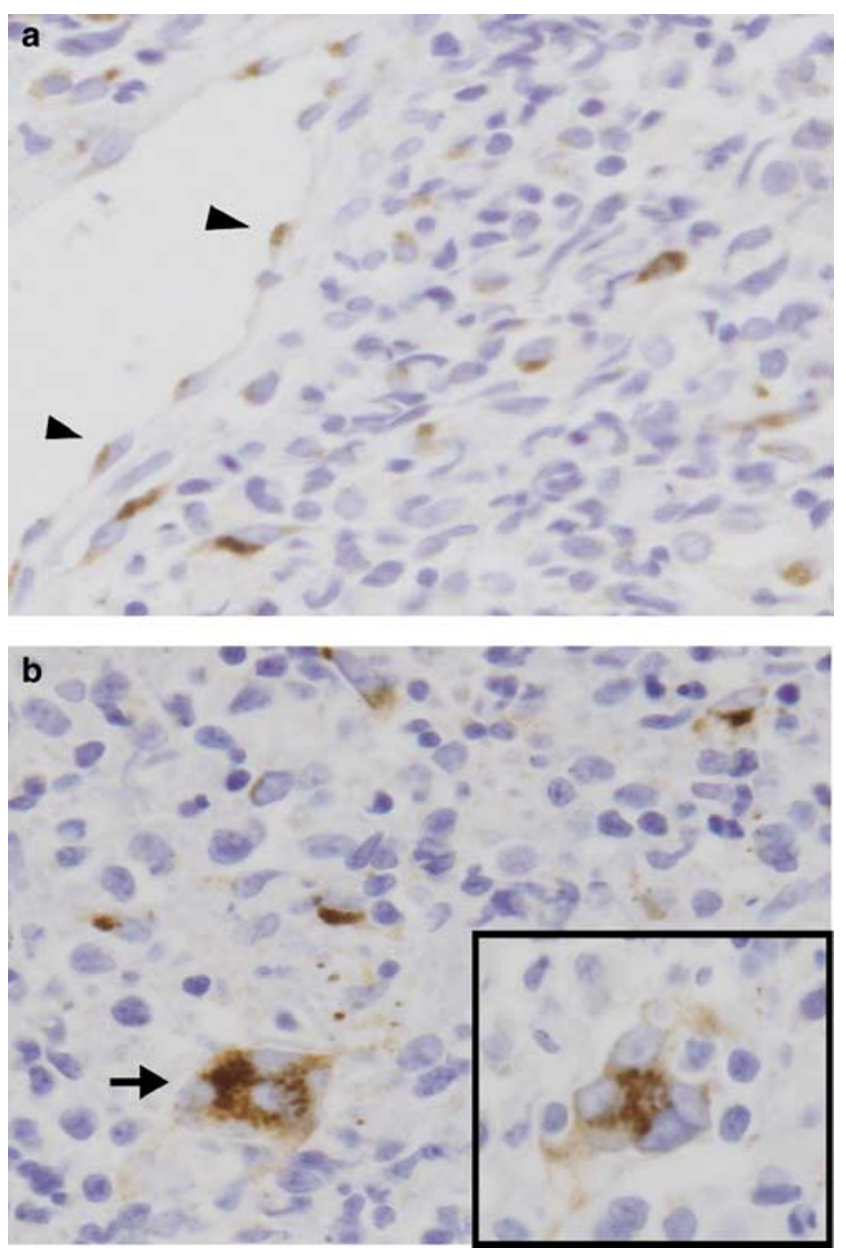

Figure 2 Immunohistochemical features of TIMP-1(+) diffuse large B-cell lymphoma. (a) TIMP-1 expression is found in stromal cells including endothelial cells (arrowheads) and fibroblasts. (b) In particular, lymphoma tumor cells with anaplasia or multinucleate nuclei (arrow, inset) frequently express high level of TIMP-1 with a Golgi staining pattern.

\section{Prognostic Significance of TIMP-1(+) Diffuse Large B-Cell Lymphoma}

Clinical data of all patients were available for survival analysis. The median follow-up of surviving patients was 12 months (range, 0.5-120 months). Univariate analysis revealed that an unfavorable overall survival was correlated with an advanced clinical stage $(P<0.001)$, IPI score $(P<0.001)$, and nongerminal center B-cell type $(P=0.017)$ (Figure $3 \mathrm{a}, \mathrm{b}$, and c). By contrast, CD10 expression was associated with a longer overall survival $(P=0.030)$ (Figure 3d). Other immunophenotypic markers were poorly correlated with overall survival. The survival curves according to the TIMP-1 expression were well separated, but it was not statistically significant $(P=0.22)$ (Figure 4a). In addition, the survival curves between TIMP-1 $(+)$ nongerminal center B-cell group and TIMP-1 $(+)$ germinal center B-cell or TIMP-1(-) nongerminal center B-cell groups were compared. The TIMP-1 $(+)$ nongerminal center 

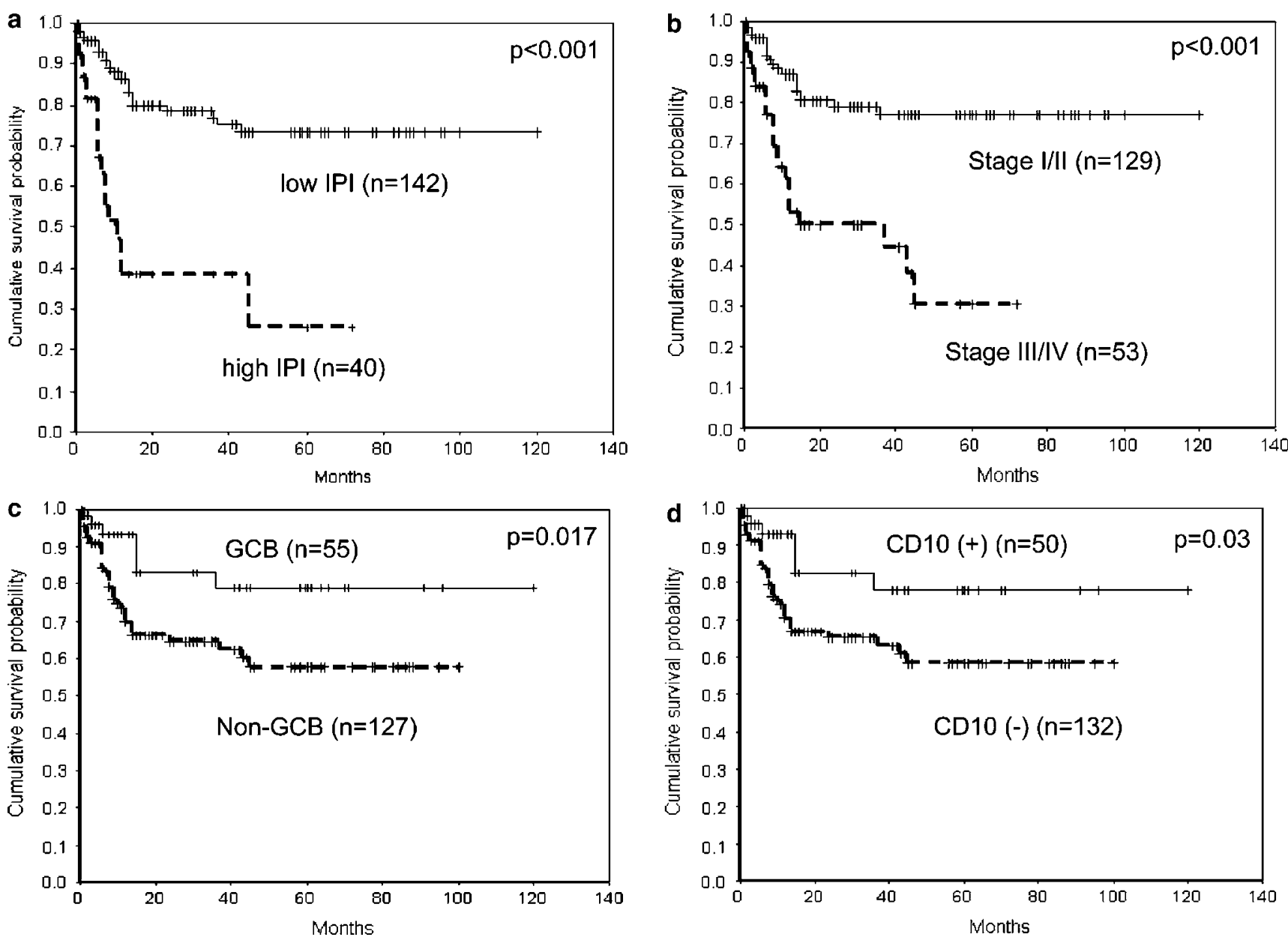

Figure 3 Kaplan-Meier survival curves of patients with diffuse large B-cell lymphoma, which are based on (a) International Prognostic Index (IPI), (b) clinical stage, (c) immunophenotypic subtype, and (d) CD10 expression.

B-cell group showed a tendency of poor survival than the TIMP-1 $(+)$ germinal center B-cell (log-rank test, $P=0.14)$ or TIMP-1(-) nongerminal center B-cell groups (log-rank test, $P=0.13$ ), respectively.

To control confounding variables and find more independent survival factors, multivariate analysis using the Cox proportional hazard model was performed. The multivariate survival analysis revealed that TIMP-1 expression in tumor cells was an independent predictor of poor overall survival with a 2.5 -fold risk ratio $(P=0.049)$ (Figure $4 \mathrm{~b}$ and Table 3$)$. The discrepancy between impacts of TIMP-1 expression on survival in univariate and multivariate analyses suggests that there are confounding variables. Generally, the variables less than 0.25 of $P$-value after univariate analysis are selected and tested for the multivariate analysis to reduce the possibility of dropping significant variables out. Moreover, since the 95\% confidence interval (1.01-6.21) of TIMP-1 expression in Cox regression analysis does not contain 1, the odd ratio was considered statistically significant (Table 3). The multivariate analysis was performed using 182 patients with complete information for all variables. The variables included: age and sex of patients, serum level of LDH, performance status, presence of $\mathrm{B}$ symptom, primary sites (nodal vs extranodal), clinical stage, IPI scores, CD10, BCL6, MUM-1, CD138, TIMP-1 and EBV infection. Among these, older age, the presence of $B$ symptoms, abnormal serum LDH, more advanced clinical stage (III/IV), and positive TIMP-1 expression were related to a shortened overall survival in the patients with diffuse large B-cell lymphoma (Table 3).

\section{Discussion}

In this study, we found that TIMP-1 is expressed not only in lymphoma cells themselves but also in stromal cells like fibroblasts and endothelial cells lining sinusoids and capillaries. In multivariate analysis, TIMP-1 expression was highly correlated with unfavorable outcomes in the patients with diffuse large B-cell lymphoma. TIMP-1 $(+)$ diffuse large B-cell lymphoma has a tendency to develop from extranodal sites such as stomach, palatine tonsils, and nasopharynx. However, TIMP-1 expres- 

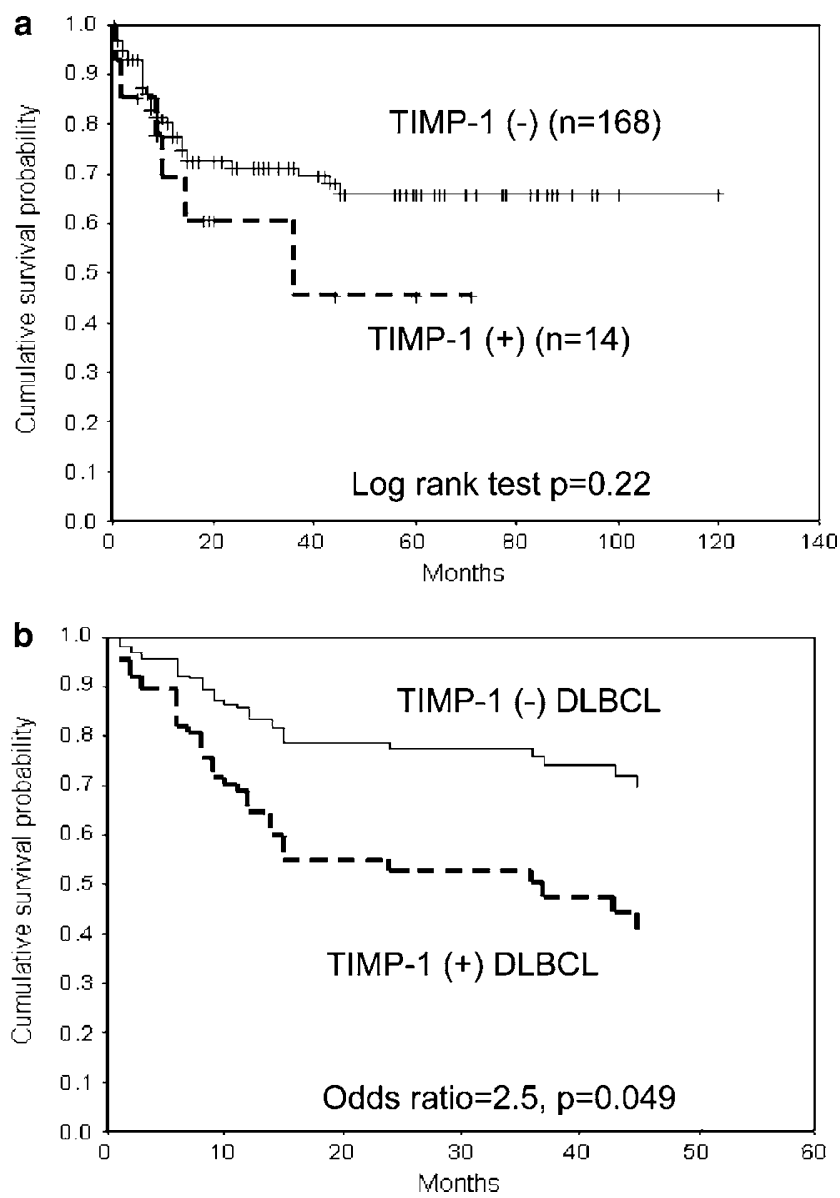

Figure 4 Survival curves of patients with diffuse large B-cell lymphoma by TIMP-1 expression. (a) Kaplan-Meier analysis shows that TIMP-1 is poorly correlated with patient survival. However, (b) multivariate analysis by Cox proportional hazard model reveals that TIMP- 1 is an independent negative prognostic factor in diffuse large B-cell lymphoma cases.

Table 3 Prognostic factors affecting overall survival by multivariate analysis

\begin{tabular}{llcc}
\hline \multirow{2}{*}{ Variables } & \multirow{2}{*}{ Unfavorable factor } & \multicolumn{2}{c}{ Multivariate } \\
\cline { 3 - 4 } & & Odds ratio (95\% CI) & $\mathrm{P}$ \\
\hline Age & $>60$ years & $2.76(1.44-5.29)$ & 0.002 \\
B symptoms & Present & $2.01(1.08-3.72)$ & 0.027 \\
Serum LDH & > Normal & $2.88(1.47-5.64)$ & 0.002 \\
Stage & III/IV & $2.27(1.18-4.40)$ & 0.015 \\
TIMP-1 & Positive & $2.50(1.01-6.21)$ & 0.049 \\
\hline
\end{tabular}

CI, confidence interval.

sion was poorly correlated with any of the subtypes of diffuse large B-cell lymphoma defined by immunohistochemistry or the status of EBV infection. Our study suggests that TIMP-1 expression is a marker for poor prognosis in patients with diffuse large B-cell lymphoma, independent of prior clinical determinants including: advanced age, the presence of B-symptoms, abnormal serum LDH, and more advanced clinical stages.

Although a de novo diffuse large B-cell lymphoma cell line with a high level of TIMP-1 has been reported, ${ }^{29}$ we first demonstrated that TIMP-1 was expressed in the lymphoma tumor cells themselves in approximately $8 \%$ of diffuse large B-cell lymphoma cases. This finding confirms prior observations using in situ hybridization. ${ }^{4}$ It is of great interest that TIMP-1 is frequently expressed in tumor cells with multinucleate or anaplastic nuclei of diffuse large B-cell lymphoma, and that TIMP-1 is highly expressed in anaplastic lymphoma kinase (ALK)-positive anaplastic large cell lymphoma by upregulation of the activation of STAT3. ${ }^{23,30}$ Moreover, it has been shown that upregulation of TIMP-1 expression, in a BL cell line, is related to the activation of STAT3. ${ }^{3}$ It has been shown that TIMP-1 is expressed in Reed-Sternberg cells of Hodgkin lymphoma ${ }^{31,32}$ and subsets of high grade B-cell lymphoma., ${ }^{4,5}$ More recently, the existence of a gray zone between diffuse large B-cell lymphoma and Hodgkin lymphoma has been proposed..$^{33}$ Since mediastinal large B-cell lymphoma and T-cell/histocyte-rich B-cell lymphoma share characteristics of Hodgkin lymphoma and diffuse large B-cell lymphoma, they are considered gray zone lymphomas. ${ }^{15,33}$ It is noteworthy that TIMP-1 expression is frequently seen in large anaplastic or multinucleate bizarre tumor cells, and has been shown to be closely related to the activation of STAT3 signaling pathway. Thus, although TIMP-1(+) diffuse large B-cell lymphoma remains to be further defined, it is speculated that it might represent a gray zone lymphoma between diffuse large B-cell lymphoma and Hodgkin lymphoma, or an anaplastic variant of diffuse large B-cell lymphoma. On the other hand, it has been reported that TIMP-1 can be translocated to the nuclei of gingival fibroblasts or breast carcinoma cells, suggesting unknown nuclear functions. ${ }^{34,35}$ However, our study found that TIMP-1 is localized only in the cytoplasm of tumor or stromal cells with distinct Golgi staining pattern. This supports the notion that TIMP-1 is produced by neoplastic cells and secreted into their microenvironment, possibly forming an autocrine/paracrine loop.

Our in vivo study revealed that TIMP-1 expression in diffuse large B-cell lymphoma is poorly correlated with the presence or absence of EBV infection. However, TIMP-1 has been shown to be associated with EBV latency II/III infection of BL cell lines in vitro. ${ }^{8}$ Hodgkin lymphoma has been known to express TIMP-1 and EBV has been detected in approximately $40 \%$ of Hodgkin lymphoma. However, no direct evidence that the R-S cells infected by EBV express TIMP-1 in vitro has been demonstrated. Recently, it has been shown that EBVencoded latent membrane protein-1 (LMP-1) can upregulate MMP- $1,{ }^{36}$ or MMP-9 by activating the NF- $\kappa \mathrm{B}$ signaling pathway, ${ }^{37,38}$ but downregulate reversion-inducing-cysteine-rich protein with Kazal 
motifs (RECK) via the activation of ERK/Sp1 signaling pathway. ${ }^{39}$ The RECK encodes a GPI-anchored glycoprotein harboring three protease inhibitor-like domains. Like TIMPs, the RECK protein regulates at least three members of the MMP family including MMP-2, MMP-9, and MT1-MMP, in vitro or in cultured cells. ${ }^{40}$ However, unlike MMP-1, MMP-9, and RECK, any evidence on the regulation of TIMP-1 expression by EBV has not yet been demonstrated. Approximately $10 \%$ of all diffuse large B-cell lymphoma studied harbored EBV, but no clinical and immunophenotypic differences between EBVpositive and -negative groups were observed. In fact, there was no significant difference in the patient outcomes between the two groups.

In vitro microarray analysis of TIMP-1-transfected BL cell lines demonstrated that TIMP-1 induces post-germinal center or plasmablastic differentiation of germinal center B-cells. ${ }^{3}$ The gene expression profiles of the nongerminal center B-cell-type diffuse large B-cell lymphoma mostly corresponded with those of post-germinal center B-cell cells. ${ }^{12}$ However, the immunophenotypes of TIMP-1 $(+)$ diffuse large B-cell lymphoma were inconsistent with the immunophenotypes of post-germinal center B cells. This finding suggests that TIMP-1 $(+)$ diffuse large B-cell lymphoma might be derived from either germinal center or post-germinal center B cells.

Our results from the multivariate analysis show that TIMP-1 is an independent marker for a poor prognosis in patients with diffuse large B-cell lymphoma. TIMP-1 is a $28.5 \mathrm{kDa}$ glycoprotein that forms noncovalent 1:1 stoichiometric complexes with MMPs, thereby inhibiting the proteolytic activity of these enzymes. ${ }^{41,42}$ Upregulation of TIMP-1 expression, at both mRNA and protein levels, has been demonstrated in several types of cancer, and has been associated with a poor prognosis in the cancer patients. ${ }^{43-47}$ Given its original role of MMP inhibition, TIMP-1 has paradoxically emerged as a poor prognostic factor and serum marker for many epithelial cancers. ${ }^{6,7}$ Thus, TIMP-1 is a multifunctional protein having growth promotion and antiapoptotic effects as well as MMP inhibitory functions.

Although the mechanisms of TIMP-1 affecting the unfavorable outcomes are poorly understood, the antiapoptotic effect of TIMP-1 may be responsible for the poor survival of cancer patients. Two different mechanisms, MMP-dependent or MMPindependent, have been proposed for the antiapoptotic function of TIMP-1. Guedez et $a l^{8}$ showed that the antiapoptotic effect of TIMP-1 was not a result of inhibition of MMPs because N-terminal alanine appended Ala + TIMP-1, which is devoid of MMP inhibitory activity, could also suppress apoptosis. In addition, TIMP-1 overexpression inhibits apoptosis even after the loss of cell adhesion in human breast epithelial MCF10A cells, suggesting that the antiapoptotic effect of TIMP-1 does not depend on its ability to stabilize cell-matrix interactions. ${ }^{48}$ Thus, it is hypothesized that antiapoptosis occurs via binding of TIMP-1 to a putative cell-surface receptor, independent of its MMP inhibitory function.

In an MCF10A breast epithelial cell line, TIMP-1 was shown to have oncogenic activity by inhibiting intrinsic and extrinsic apoptosis and by disrupting lumen formation. ${ }^{49,50}$ Overexpression of TIMP-1 induces constitutive activation of focal adhesion kinase (FAK) through tyrosine phosphorylation..$^{48,49}$ FAK is an upstream regulator of phosphatidylinositol-3 kinase (PI-3 kinase), leading to regulation of BCL-2 family members and cell survival. On the other hand, in a breast carcinoma cell line, the antiapoptotic effect of TIMP-1 is mediated by the sequential activation of pertussis toxin-sensitive G protein, c-Src, PI-3 kinase, and Akt. ${ }^{51}$ In BL cells, overexpression of TIMP-1 leads to the activation and expression of STAT3, and upregulates the expression of BCL- $\mathrm{X}_{\mathrm{L}}$, cyclin D2, and CD44. ${ }^{3,52}$ Interestingly, BCL- $\mathrm{X}_{\mathrm{L}}$ is a target protein of activated STAT3 and plays a major role of antiapoptosis. ${ }^{53}$ CD44 is also known to promote cell survival by activating the PI-3 kinase/Akt pathway. ${ }^{54-57}$ The expression of cyclin D2 or CD44 is also correlated with a poor prognosis in patients with diffuse large B-cell lymphoma. ${ }^{19,58}$ Therefore, although there is discrepancy in TIMP-1 signaling pathways depending on cell type,TIMP-1 appears to inhibit apoptosis by activating the PI-3 kinase/Akt and/or STAT3 signaling cascades. The activation of these signaling pathways regulates BCL-2 family members, especially involving $B C L-\mathrm{X}_{\mathrm{L}}$ resulting in inhibition of caspase activation and thereby inhibition of apoptosis.

We analyzed unsupervised hierarchical clustering using the correlation coefficient with centering; a similarity measure that is sensitive to the expression profile shape, regardless of the expression levels. ${ }^{28}$ As a result, TIMP-1 was completely separated from the other clusters of phenotypic markers or EBV infection status. This suggests that TIMP-1 $(+)$ diffuse large B-cell lymphoma is a distinct group separate from other cases of diffuse large B-cell lymphoma. However, the expression of CD138 or TIMP1 as well as EBV infection appears to be relatively rare events in diffuse large B-cell lymphoma. In the multivariate survival analysis, older age, the presence of B-symptoms, abnormal serum LDH, more advanced clinical stage (III/IV), and TIMP-1 expression were related to poorer overall survival in the patients with diffuse large B-cell lymphoma. This result is in agreement with those of previous studies of diffuse large B-cell lymphoma using tissue microarray, ${ }^{59-62}$ reflecting the reliability and robustness of our data. Therefore, TIMP-1 expression is an independent factor, and represents a marker for poor prognosis with potential therapeutic implications. However, identification of a putative cell surface receptor for TIMP-1 and its downstream signaling pathways require further study. 
In conclusion, this study clearly shows that TIMP1 expression is an independent factor associated with a poor prognosis in patients with diffuse large B-cell lymphoma. Thus, TIMP-1 may play a crucial role in the tumor progression of diffuse large B-cell lymphoma rather than define the histogenetic origin of diffuse large B-cell lymphoma or EBV infection status. Further, it is suggested that TIMP-1 could represent a novel therapeutic target for a subset of diffuse large B-cell lymphoma.

\section{Acknowledgements}

We thank Sang-Ju Lee, Mi-Ran Jeong, Eun-Ja Lee, Sung-Su Lee, and Jee-Hye Ok for their technical assistance.

\section{References}

1 Hayakawa T, Yamashita K, Tanzawa K, et al. Growthpromoting activity of tissue inhibitor of metalloproteinases-1 (TIMP-1) for a wide range of cells. A possible new growth factor in serum. FEBS Lett 1992;298: 29-32.

2 Khokha R. Suppression of the tumorigenic and metastatic abilities of murine B16-F10 melanoma cells in vivo by the overexpression of the tissue inhibitor of the metalloproteinases-1. J Natl Cancer Inst 1994;86: 299-304.

3 Guedez L, Martinez A, Zhao S, et al. Tissue inhibitor of metalloproteinase 1 (TIMP-1) promotes plasmablastic differentiation of a Burkitt lymphoma cell line: implications in the pathogenesis of plasmacytic/ plasmablastic tumors. Blood 2005;105:1660-1668.

4 Kossakowska AE, Urbanski SJ, Edwards DR. Tissue inhibitor of metalloproteinases-1 (TIMP-1) RNA is expressed at elevated levels in malignant non-Hodgkin's lymphomas. Blood 1991;77:2475-2481.

5 Kossakowska AE, Urbanski SJ, Watson A, et al. Patterns of expression of metalloproteinases and their inhibitors in human malignant lymphomas. Oncol Res 1993;5:19-28.

6 Yoshikawa T, Saitoh M, Tsuburaya A, et al. Tissue inhibitor of matrix metalloproteinase-1 in the plasma of patients with gastric carcinoma. A possible marker for serosal invasion and metastasis. Cancer 1999;86: 1929-1935.

7 Holten-Andersen MN, Stephens RW, Nielsen HJ, et al. High preoperative plasma tissue inhibitor of metalloproteinase-1 levels are associated with short survival of patients with colorectal cancer. Clin Cancer Res 2000;6:4292-4299.

8 Guedez L, Courtemanch L, Stetler-Stevenson M. Tissue inhibitor of metalloproteinase (TIMP)-1 induces differentiation and an antiapoptotic phenotype in germinal center B cells. Blood 1998;92:1342-1349.

9 The non-Hodgkin's Lymphoma Classification Project. A clinical evaluation of the International Lymphoma Study Group classification of non-Hodgkin's lymphoma. Blood 1997;89:3909-3918.

10 Coiffier B. Diffuse large cell lymphoma. Curr Opin Oncol 2001;13:325-334.
11 Rossi D, Gaidano G. Molecular heterogeneity of diffuse large B-cell lymphoma: implications for disease management and prognosis. Hematology 2002;7: 239-252.

12 Alizadeh AA, Eisen MB, Davis RE, et al. Distinct types of diffuse large B-cell lymphoma identified by gene expression profiling. Nature 2000;403:503-511.

13 Rosenwald A, Wright G, Chan WC, et al. The use of molecular profiling to predict survival after chemotherapy for diffuse large-B-cell lymphoma. N Engl J Med 2002;346:1937-1947.

14 Rosenwald A, Wright G, Leroy K, et al. Molecular diagnosis of primary mediastinal B cell lymphoma identifies a clinically favorable subgroup of diffuse large B cell lymphoma related to Hodgkin lymphoma. J Exp Med 2003;198:851-862.

15 Monti S, Savage KJ, Kutok JL, et al. Molecular profiling of diffuse large B-cell lymphoma identifies robust subtypes including one characterized by host inflammatory response. Blood 2005;105:1851-1861.

16 Kuze T, Nakamura N, Hashimoto Y, et al. The characteristics of Epstein-Barr virus (EBV)-positive diffuse large B-cell lymphoma: comparison between $\mathrm{EBV}(+)$ and $\mathrm{EBV}(-)$ cases in Japanese population. Jpn J Cancer Res 2000;91:1233-1240.

17 Hummel M, Anagnostopoulos I, Korbjuhn P, et al. Epstein-Barr virus in B-cell non-Hodgkin's lymphomas: unexpected infection patterns and different infection incidence in low- and high-grade types. J Pathol 1995;175:263-271.

18 Jaffe ES HN, Stein H, Vardiman JW (eds). World Health Organization Classification of Tumours. Pathology and Genetics of Tumours of Haematopoietic and Lymphoid Tissues. IARC Press: Lyon, 2001.

19 Hans CP, Weisenburger DD, Greiner TC, et al. Expression of PKC-beta or cyclin D2 predicts for inferior survival in diffuse large B-cell lymphoma. Mod Pathol 2005;18:1377-1384.

20 Natkunam Y, Warnke RA, Montgomery $\mathrm{K}$, et al. Analysis of MUM1/IRF4 protein expression using tissue microarrays and immunohistochemistry. Mod Pathol 2001;14:686-694.

21 Hedvat CV, Hegde A, Chaganti RS, et al. Application of tissue microarray technology to the study of nonHodgkin's and Hodgkin's lymphoma. Hum Pathol 2002;33:968-974.

22 Milanes-Yearsley M, Hammond ME, Pajak TF, et al. Tissue micro-array: a cost and time-effective method for correlative studies by regional and national cancer study groups. Mod Pathol 2002;15:1366-1373.

23 Lai R, Rassidakis GZ, Medeiros LJ, et al. Signal transducer and activator of transcription-3 activation contributes to high tissue inhibitor of metalloproteinase-1 expression in anaplastic lymphoma kinasepositive anaplastic large cell lymphoma. Am J Pathol 2004;164:2251-2258.

24 Hans CP, Weisenburger DD, Greiner TC, et al. Confirmation of the molecular classification of diffuse large B-cell lymphoma by immunohistochemistry using a tissue microarray. Blood 2004;103:275-282.

25 Liu CL, Prapong W, Natkunam Y, et al. Software tools for high-throughput analysis and archiving of immunohistochemistry staining data obtained with tissue microarrays. Am J Pathol 2002;161:1557-1565.

26 Eisen MB, Spellman PT, Brown PO, et al. Cluster analysis and display of genome-wide expression patterns. Proc Natl Acad Sci USA 1998;95:14863-14868. 
27 Ko YH, Kim CW, Park CS, et al. REAL classification of malignant lymphomas in the Republic of Korea: incidence of recently recognized entities and changes in clinicopathologic features. Hematolymphoreticular Study Group of the Korean Society of Pathologists. Revised European-American lymphoma. Cancer 1998; 83:806-812.

28 Leung YF, Cavalieri D. Fundamentals of cDNA microarray data analysis. Trends Genet 2003;19:649-659.

29 Maesako Y, Uchiyama T, Ohno H. Comparison of gene expression profiles of lymphoma cell lines from transformed follicular lymphoma, Burkitt's lymphoma and de novo diffuse large B-cell lymphoma. Cancer Sci 2003;94:774-781.

30 Rust R, Blokzijl T, Harms G, et al. TIMP-1 expression in anaplastic large cell lymphoma is usually restricted to macrophages and only seldom observed in tumour cells. J Pathol 2005;206:445-450.

31 Oelmann E, Herbst H, Zuhlsdorf M, et al. Tissue inhibitor of metalloproteinases 1 is an autocrine and paracrine survival factor, with additional immuneregulatory functions, expressed by Hodgkin/ReedSternberg cells. Blood 2002;99:258-267.

32 Pennanen H, Kuittinen O, Soini Y, et al. Clinicopathological correlations of TIMP-1 and TIMP-2 in Hodgkin's lymphoma. Eur J Haematol 2004;72:1-9.

33 Abramson JS, Shipp MA. Advances in the biology and therapy of diffuse large B-cell lymphoma: moving toward a molecularly targeted approach. Blood 2005;106:1164-1174.

34 Zhao WQ, Li H, Yamashita K, et al. Cell cycleassociated accumulation of tissue inhibitor of metalloproteinases-1 (TIMP-1) in the nuclei of human gingival fibroblasts. J Cell Sci 1998;111(Part 9):1147-1153.

35 Ritter LM, Garfield SH, Thorgeirsson UP. Tissue inhibitor of metalloproteinases-1 (TIMP-1) binds to the cell surface and translocates to the nucleus of human MCF-7 breast carcinoma cells. Biochem Biophys Res Commun 1999;257:494-499.

$36 \mathrm{Lu} \mathrm{J}$, Chua HH, Chen SY, et al. Regulation of matrix metalloproteinase-1 by Epstein-Barr virus proteins. Cancer Res 2003;63:256-262.

37 Takeshita H, Yoshizaki T, Miller WE, et al. Matrix metalloproteinase 9 expression is induced by EpsteinBarr virus latent membrane protein 1 C-terminal activation regions 1 and 2. J Virol 1999;73:5548-5555.

38 Horikawa T, Yoshizaki T, Sheen TS, et al. Association of latent membrane protein 1 and matrix metalloproteinase 9 with metastasis in nasopharyngeal carcinoma. Cancer 2000;89:715-723.

39 Liu LT, Peng JP, Chang HC, et al. RECK is a target of Epstein-Barr virus latent membrane protein 1. Oncogene 2003;22:8263-8270.

40 Noda M, Oh J, Takahashi R, et al. RECK: a novel suppressor of malignancy linking oncogenic signaling to extracellular matrix remodeling. Cancer Metast Rev 2003;22:167-175.

41 Lambert E, Dasse E, Haye B, et al. TIMPs as multifacial proteins. Crit Rev Oncol Hematol 2004;49:187-198.

42 Brew K, Dinakarpandian D, Nagase H. Tissue inhibitors of metalloproteinases: evolution, structure and function. Biochim Biophys Acta 2000;1477:267-283.

43 Ree AH, Florenes VA, Berg JP, et al. High levels of messenger RNAs for tissue inhibitors of metalloproteinases (TIMP-1 and TIMP-2) in primary breast carcinomas are associated with development of distant metastases. Clin Cancer Res 1997;3:1623-1628.
44 McCarthy K, Maguire T, McGreal G, et al. High levels of tissue inhibitor of metalloproteinase-1 predict poor outcome in patients with breast cancer. Int J Cancer 1999;84:44-48.

45 Nakopoulou L, Giannopoulou I, Stefanaki K, et al. Enhanced mRNA expression of tissue inhibitor of metalloproteinase-1 (TIMP-1) in breast carcinomas is correlated with adverse prognosis. J Pathol 2002;197: 307-313.

46 Schrohl AS, Christensen IJ, Pedersen AN, et al. Tumor tissue concentrations of the proteinase inhibitors tissue inhibitor of metalloproteinases-1 (TIMP-1) and plasminogen activator inhibitor type 1 (PAI-1) are complementary in determining prognosis in primary breast cancer. Mol Cell Proteomics 2003;2: 164-172.

47 Schrohl AS, Holten-Andersen MN, Peters HA, et al. Tumor tissue levels of tissue inhibitor of metalloproteinase-1 as a prognostic marker in primary breast cancer. Clin Cancer Res 2004;10:2289-2298.

48 Li G, Fridman R, Kim HR. Tissue inhibitor of metalloproteinase-1 inhibits apoptosis of human breast epithelial cells. Cancer Res 1999;59:6267-6275.

49 Liu XW, Bernardo MM, Fridman R, et al. Tissue inhibitor of metalloproteinase-1 protects human breast epithelial cells against intrinsic apoptotic cell death via the focal adhesion kinase/phosphatidylinositol 3-kinase and MAPK signaling pathway. J Biol Chem 2003;278:40364-40372.

50 Liu XW, Taube ME, Jung KK, et al. Tissue inhibitor of metalloproteinase-1 protects human breast epithelial cells from extrinsic cell death: a potential oncogenic activity of tissue inhibitor of metalloproteinase-1. Cancer Res 2005;65:898-906.

51 Lee SJ, Yoo HJ, Bae YS, et al. TIMP-1 inhibits apoptosis in breast carcinoma cells via a pathway involving pertussis toxin-sensitive $\mathrm{G}$ protein and c-Src. Biochem Biophys Res Commun 2003;312:1196-1201.

52 Guedez L, Stetler-Stevenson WG, Wolff L, et al. In vitro suppression of programmed cell death of B cells by tissue inhibitor of metalloproteinases-1. J Clin Invest 1998;102:2002-2010.

53 Hsieh FC, Cheng G, Lin J. Evaluation of potential Stat3regulated genes in human breast cancer. Biochem Biophys Res Commun 2005;335:292-299.

54 van der Voort R, Keehnen RM, Beuling EA, et al. Regulation of cytokine signaling by $\mathrm{B}$ cell antigen receptor and CD40-controlled expression of heparan sulfate proteoglycans. J Exp Med 2000;192:1115-1124.

55 Bates RC, Edwards NS, Burns GF, et al. A CD44 survival pathway triggers chemoresistance via lyn kinase and phosphoinositide 3-kinase/Akt in colon carcinoma cells. Cancer Res 2001;61:5275-5283.

56 Lin YH, Yang-Yen HF. The osteopontin-CD44 survival signal involves activation of the phosphatidylinositol 3-kinase/Akt signaling pathway. J Biol Chem 2001;276: 46024-46030.

57 Lakshman M, Subramaniam V, Rubenthiran U, et al. CD44 promotes resistance to apoptosis in human colon cancer cells. Exp Mol Pathol 2004;77:18-25.

58 Tzankov A, Pehrs AC, Zimpfer A, et al. Prognostic significance of CD44 expression in diffuse large B cell lymphoma of activated and germinal centre B cell-like types: a tissue microarray analysis of 90 cases. J Clin Pathol 2003;56:747-752.

59 Biasoli I, Morais JC, Scheliga A, et al. CD10 and Bcl-2 expression combined with the International Prognostic 
Index can identify subgroups of patients with diffuse large-cell lymphoma with very good or very poor prognoses. Histopathology 2005;46:328-333.

60 Chang CC, McClintock S, Cleveland RP, et al. Immunohistochemical expression patterns of germinal center and activation B-cell markers correlate with prognosis in diffuse large B-cell lymphoma. Am J Surg Pathol 2004;28:464-470.
61 Jerkeman M, Anderson H, Dictor M, et al. Assessment of biological prognostic factors provides clinically relevant information in patients with diffuse large B-cell lymphoma-a Nordic Lymphoma Group study. Ann Hematol 2004;83:414-419.

62 Moller MB, Pedersen NT, Christensen BE. Factors predicting long-term survival in low-risk diffuse large B-cell lymphoma. Am J Hematol 2003;74:94-98. 